\title{
An efficient and accurate approach for fabricating dental implant surgical guides
}

\author{
Wei-chen Lee*, Ching-Hang Huang, Shan-Chen Chung and Ching-chih Wei \\ Department of Mechanical Engineering, National Taiwan University of Science and \\ Technology, Taipei 10607, Taiwan, Republic of China
}

\begin{abstract}
The aim of this research was to develop an efficient and accurate method to fabricate a dental implant surgical guide. The surgical guide is adapted from the patient's vacuum-formed clear template with the use of a plate with three ceramic balls, a six-axis drilling machine and its fixture. The plate, with the ceramic balls used as radiographic markers, is glued to the template, and the patient bites this template during a CT scan. Then, the surgeon can plan the locations and orientations of the implants on the CT-based model in the dental planning software. The drilling information is exported directly to the computer-controlled drilling machine for subsequent drilling on the template to complete the surgical guide. This method allows the surgical guide to be made without any measurements, which reduces the fabrication time, but increases the drilling accuracy. The preliminary results show that the average location error was $0.31 \pm 0.17 \mathrm{~mm}$ and the average orientation error was $0.53 \pm 0.24^{\circ}$, which can be considered accurate in comparison with the results reported in the literature.
\end{abstract}

Keywords: Dental implant surgical guide, drilling machine, radiographic marker, fixture, vacuum-formed template

\section{Introduction}

With the high satisfaction rate of dental implant patients, dental implantation has become a popular procedure for edentulous people. To increase the success rate, many researchers have adopted experimental or numerical methods [1,2] so as to ensure the proper life and stress tolerance of the implant. In addition to studying the implant itself, the proper location and orientation of the implant are also critical considerations. To achieve this desired result, tools are needed to help surgeons drill accurately during dental implant surgery. One of the common tools is the dental implant surgical guide.

A surgical guide, usually made of polymer, is supported by the patient's teeth, mucosa or bone. There are pre-drilled holes on the surgical guide and, during the dental implant surgery, the surgeon uses these holes to guide the drill bit when drilling the holes at the desired locations and orientations on the patient's maxilla or mandible for the subsequent implantation. Using the surgical guide can greatly reduce the risks and the time required for the dental implant surgery, thus increasing the success rate of dental implantation.

\footnotetext{
${ }^{*}$ Corresponding author: Wei-chen Lee, Department of Mechanical Engineering, National Taiwan University of Science and Technology, Taipei 10607, Taiwan, Republic of China. Tel.: +886-(0)2-2737-6478; Fax: +886-(0)2-2737-6460; E-mail: wclee@mail.ntust.edu.tw.
} 
Some surgical guides are simply made by using a drill press with the help of the surveyor to manually control the orientation during drilling. For example, Becker et al. [3], Atsu [4] and Arfai and Kiatamnuay [5] adopted this method for drilling holes on vacuum-formed templates in order to create surgical guides. Similarly, Akca et al. [6], Wat et al. [7] and George et al. [8] used autopolymerizing acrylic resin to make surgical guides. Shotwell et al. [9] used a desktop drill press to drill holes in the cast, inserting drill blanks in the holes and then using light-polymerizing tray material to form the surgical guide over the cast. A surgical guide made in this way is usually convenient, but the locations and orientations may not be optimal.

Another major method when making surgical guides is that using computer-aided design/computeraided manufacture (CAD/CAM) technology, and these surgical guides are usually referred to as stereolithographic surgical guides. Stereolithography, also known as rapid-prototyping (RP) or 3D printing, is an additive manufacturing method of stacking material layer-by-layer. To employ this technique, a CT scan of the patient is first required in order to create a three-dimensional computer model, which the surgeon then uses to plan the locations and the orientations of the implants. The model is then transferred to an RP machine or a 3D printer in order to fabricate the surgical guide by using stereolithography. Some of the studies on surgical guides made using stereolithography can be found in $[10,11]$. The stereolithographic surgical guide offers less risk in the consideration of the patient's anatomical structure during the planning stage. However, the fabrication time using the RP machine may take hours. In addition, the completed stereolithographic surgical guide cannot be modified. Accuracy may be another potential issue, as the RP product may deform or warp due to the residual stresses, which may affect the accuracy of the locations and the orientations of the holes on the surgical guide.

The aim of this research was to propose an innovative method with the use of CAD/CAM technology to fabricate a dental implant surgical guide based on the patient's vacuum-formed clear template. This method, which used a computer-controlled drilling machine instead of an RP machine, allowed the surgical guide to be fabricated efficiently, without sacrificing accuracy or increasing the risk. The objectives of this research were, first, to use three points on the surgical guide to build a coordinate system that could be referred to in the CT images as needed, and, second, to always position the three points at the same locations with the help of the fixture of the drilling machine. As a result, no measurements were needed for the locations of the three points, thus reducing measurement error, while at the same time improving efficiency and accuracy.

\section{Method}

The method proposed in this paper integrated a computer-controlled drilling machine with a specially-designed fixture and a dental planning software package. A vacuum-formed clear template was adopted as the basis of the surgical guide, and radiographic markers were attached to the vacuumformed template so that the planned positions and orientations in the dental planning software could be calculated, based on the coordinate systems defined by the radiographic markers. The procedure required to make the surgical guide is briefly outlined as follows.

The first step is to make a vacuum-formed clear template over the patient's cast by using a pressure molding machine. Next, three ceramic balls, used as the radiographic markers, are attached to the template in a consistent way. Then, the patient is instructed to clench the template as the CT scan is being taken. The CT scan enables the three-dimensional computer model to be built with the use of the dental planning software. The surgeon can then plan the locations and orientations of the implants and transfer this information to the computer-controlled drilling machine for the subsequent drilling on the 


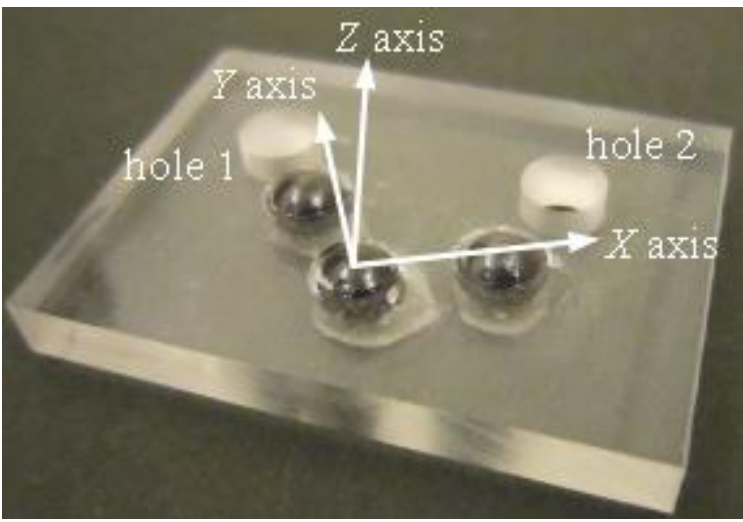

(a)

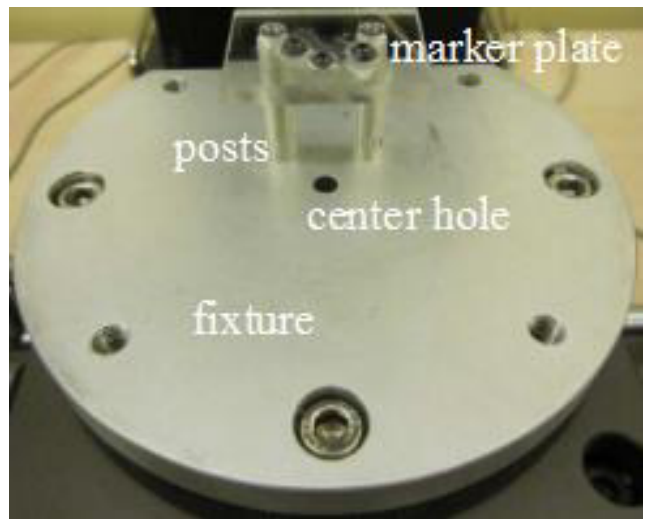

(b)

Fig. 1. (a) Marker plate with three ceramic balls; (b) marker plate screwed onto the two posts of the fixture.

vacuum-formed template, which completes the surgical guide. The details of these important steps are now explained.

The implant planning information is based on the coordinate system called the ceramic-ball coordinate system. The coordinate system is defined by the centers of the three ceramic balls. The vacuumformed template with the ceramic balls is screwed onto the fixture of the drilling machine. With deliberate design, the coordinates of the three ceramic balls, with respect to the machine's own coordinate system, are predetermined, making it easy to convert the drilling information based on the ceramicball coordinate system to the machine's coordinate system. The three ceramic balls, the drilling machine and its fixture are critical components for fabricating the surgical guide precisely and accurately.

The three ceramic balls (Chena Enterprise, Taiwan), $4 \mathrm{~mm}$ in diameter, are attached to an acrylic resin plate by placing them into the pre-drilled holes and using super glue to fix them into position, as shown in Figure 1(a), completing what is called the marker plate in this paper. The ceramic balls are used as radiographic markers, because their centers in the CT images are used to define a Cartesian coordinate system, the ceramic-ball coordinate system for the planned position and orientation of the implant. The center of the middle ball in Figure 1(a) is set as the origin; the origin to the center of the right ball defines the $X$ axis and the centers of the three ceramic balls define the $X Y$ plane. It follows that the $Z$ axis can be defined as the vertical direction of the $X Y$ plane and the $Y$ axis can be defined as the outer product of the $Z$ axis and the $X$ axis, thereby completing the ceramic-ball coordinate system. The two holes on the plate in Figure 1(a) are used for attaching it to the two posts of the fixture associated with the drilling machine, as shown in Figure 1(b). A hole in the center of the fixture can be observed in Figure 1(b). The hole and the top surface of the fixture are used to align the drill bit.

Next, the vacuum-formed template is glued to the marker plate, as shown in Figure 2(a). Figure 2(b) shows the CT image of the template with the marker plate on the cast. In reality, the patient needs to wear the template while the CT scan is being taken. In this research, the implant planning software used was Simplant Max (Saturn Inc., Taiwan). This software imports the DICOM data of the CT images and converts it to a three-dimensional model, so the surgeon can plan the implant location and orientation, and export the drilling information in terms of the ceramic-ball coordinate system. Because the positions of the holes and ceramic balls on the marker plate, as shown in Figure 1(a), are known by a one-time measurement, and the two holes on the marker plate are tightly fit to the two posts of the fixture, the coordinates of the three ceramic balls, in terms of the machine's coordinate system, can be obtained, as long as the fixture's location relative to the drilling machine's coordinate 


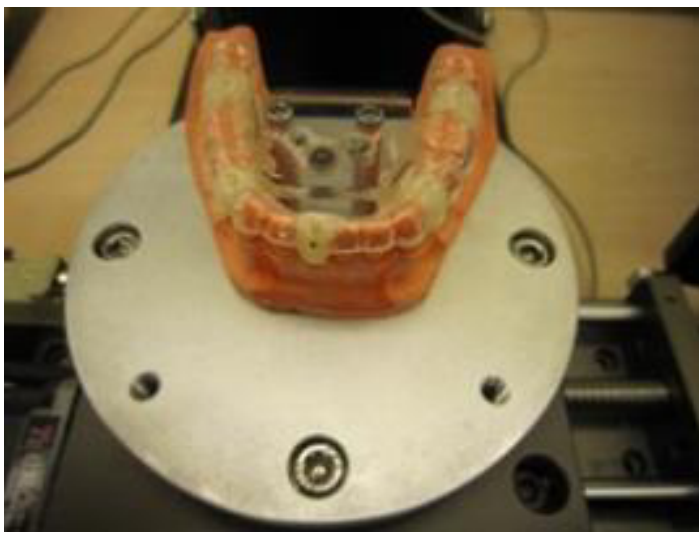

(a)

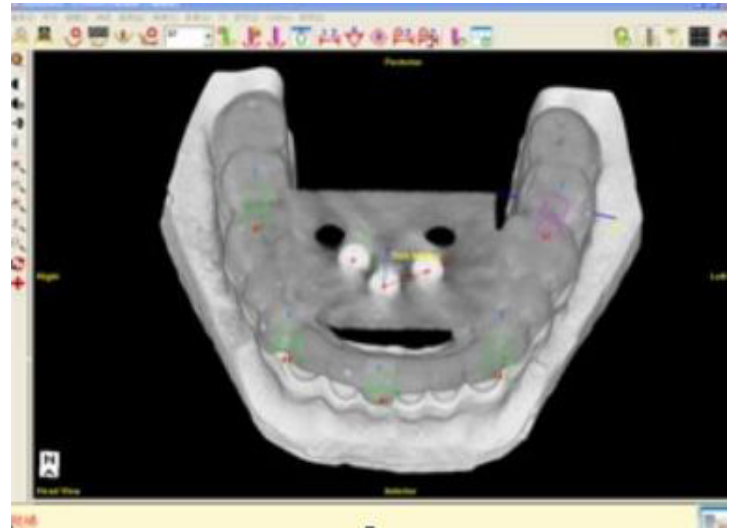

(b)

Fig. 2. (a) Vacuum-formed template placed on the cast is glued to the marker plate attached to the two posts of the fixture; (b) CT image of the template with the marker plate on the cast.

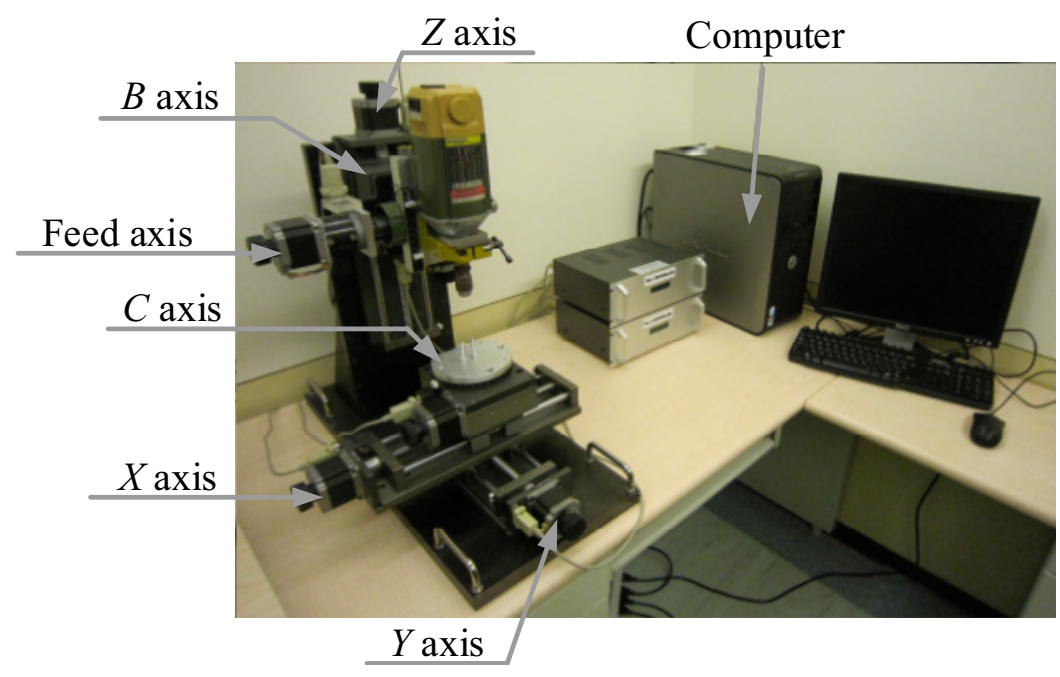

Fig. 3. Self-developed six-axis drill machine controlled by a computer.

system is known, which can also be achieved by a one-time measurement. It follows that the drilling information, in terms of the ceramic-ball coordinate system in the dental planning software, can be easily converted to the drilling locations and orientations in terms of the machine's coordinate system. Then, the holes on the vacuum-formed template can be drilled, as planned in the dental planning software, to complete the template-based surgical guide. Patients' vacuum-formed templates are all different. However, as long as the marker plate with the same design, as shown in Figure 1(a), is glued to the various vacuum-formed templates, there is no need to measure either the positions of the radiographic markers or the centers of the three ceramic balls for each template, because the positions are predetermined by design. Doing so reduces both construction time and measurement error. Therefore, the marker plate and the fixture of the drilling machine are key to the success of the proposed method.

The drilling machine used in this research was a self-developed six-axis computer-controlled machine, as shown in Figure 3. The drill was obtained from a commercial milling/drilling system (Proxx- 
on, Germany). For the six-axis drilling machine, the three linear axes are $X, Y$ and $Z$ axes, which are perpendicular to each other; the two rotational axes are $B$ and $C$ axes. The rotational $B$ axis rotates about the $Y$ axis and the rotational $C$ axis rotates about the $Z$ axis. The sixth axis, the feed axis, allows the drill to move up and down to complete the drilling process. By using this six-axis drilling machine, the holes on the surgical guide can be drilled at any location and angle within the workspace, because the three linear axes can move the drill bit to any location and the two rotational axes can control the drill bit to any desired orientation.

\section{Results and discussion}

Both a model study and a clinical study were performed to evaluate the accuracy of the proposed method. The model study started with a CT scan of the model as shown in Figure 4. After the CT images had been converted to the three-dimensional model on the computer, the surgeon planned the drilling locations and orientations, and then exported the information to the computer-controlled drilling machine. The fabrication time for drilling holes on the vacuum-formed template was less than three minutes per hole. For a surgical guide with five holes for five implants, the construction time after planning was less than 15 minutes. If the fabrication time for the vacuum-formed template and the time for gluing the radiographic marker plate to the template were included, the total construction time for a surgical guide made using the proposed method would be about 30 minutes or less, rather than the hours usually required to make a stereolithographic surgical guide. However, the use of the proposed method to fabricate surgical guides involves a learning curve. More handwork is involved in the procedure, so the proposed method is not as automatic as the method using a 3D printer.

After drilling with a 2-mm drilling bit, an aluminum tube was inserted into each hole of the surgical guide, and a CT scan was performed again with the surgical guide fixed on the cast. The aluminum tubes seen in the CT images indicated the actual drilling positions. The post-drilling CT image was fused with the image of the surgical planning, as shown in Figure 5, to calculate the deviation between the planned and the actual drilling positions and orientations. In this model study, five holes were drilled on one model; the results are summarized in Table 1. According to the preliminary evaluation results, the mean errors of the locations and angles of the drilled holes were $0.42 \mathrm{~mm}$ and $0.49^{\circ}$, respectively, which indicated that the method presented in this paper was both correct and feasible.

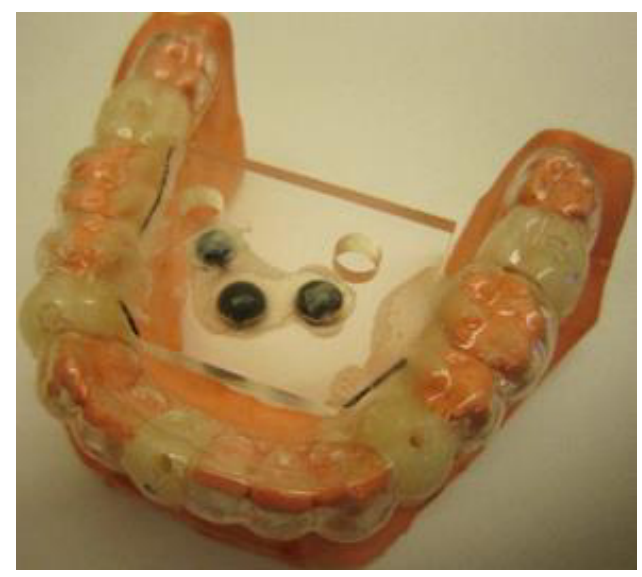

Fig. 4. Model used in the study of accuracy. 


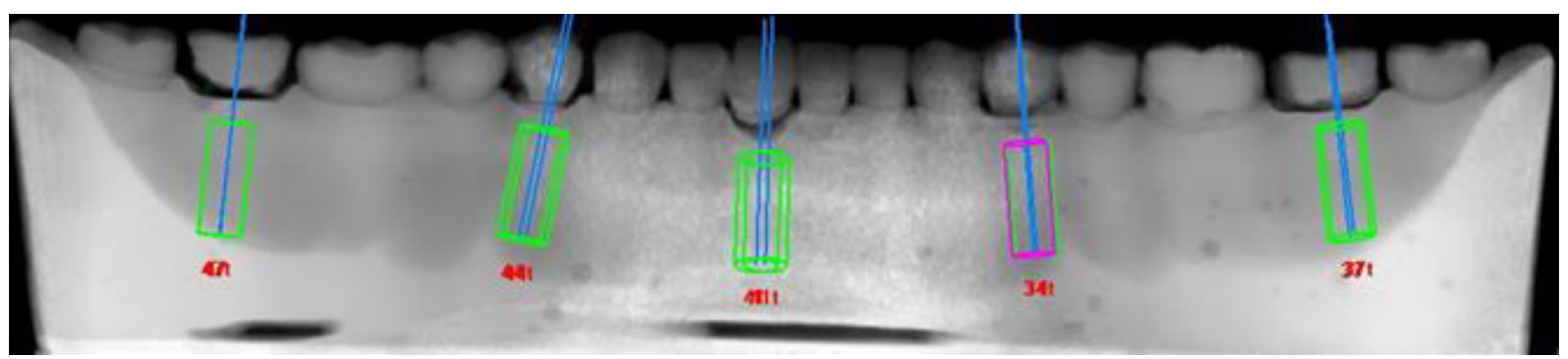

Fig. 5. Fused images of the planned and the actual holes.

Table 1

Deviation in position and orientation between the planned versus actual holes in the model study

\begin{tabular}{llllllllllll}
\hline Position & 37 & & 34 & & 41 & \multicolumn{2}{c}{44} & \\
\hline & Coronal & Angular & Coronal & Angular & Coronal & Angular & Coronal & Angular & Coronal & Angular \\
\hline & $(\mathrm{mm})$ & $\left(^{\circ}\right)$ & $(\mathrm{mm})$ & $\left(^{\circ}\right)$ & $(\mathrm{mm})$ & $\left(^{\circ}\right)$ & $(\mathrm{mm})$ & $\left(^{\circ}\right)$ & $(\mathrm{mm})$ & $\left({ }^{\circ}\right)$ \\
\hline Deviation & 0.42 & 0.91 & 0.31 & 0.66 & 0.66 & 0.20 & 0.53 & 0.10 & 0.16 & 0.56 \\
\hline Average & 0.42 & 0.49 & & & & & & & & \\
\hline
\end{tabular}

For the clinical study, four patients were involved. Eight implants in total were placed using surgical guides fabricated by the proposed method in order to evaluate the accuracy of the surgical guide. After the fusion of the preoperative and postoperative images, a variation was observed of $0.31 \pm 0.17$ $\mathrm{mm}($ mean $\pm \mathrm{SD})$ in the coronal deviation and $0.53 \pm 0.24^{\circ}$ in the angular deviation as listed in Table 2. Considering the limited number of samples, the results may not be significant. However, by comparing our results to those reported in [12], where the deviation was $0.9 \pm 0.5 \mathrm{~mm}$ at the implant head and the deviation of the angle was $4.5 \pm 2^{\circ}$ using stereolithographic surgical guides, the accuracy of the fabrication method of surgical guides as presented in the paper could be confirmed.

Table 2

Deviation in position and orientation between the planned versus actual implants in the clinical study

\begin{tabular}{lllllll}
\hline & 1st Placement & $2^{\text {nd }}$ Placement & \multicolumn{3}{c}{$3^{\text {rd }}$ Placement } \\
\hline Patient & $\begin{array}{l}\text { Coronal Angular } \\
(\mathrm{mm})\end{array}$ & $\begin{array}{l}\text { Coronal } \\
(\mathrm{mm})\end{array}$ & $\begin{array}{l}\text { Angular } \\
\left({ }^{\circ}\right)\end{array}$ & $\begin{array}{l}\text { Coronal } \\
(\mathrm{mm})\end{array}$ & $\begin{array}{l}\text { Angular } \\
\left({ }^{\circ}\right)\end{array}$ \\
\hline 1 & 0.27 & 0.41 & 0.23 & 0.26 & 0.05 & 0.97 \\
\hline 2 & 0.11 & 0.45 & 0.44 & 0.28 & & \\
\hline 3 & 0.51 & 0.61 & 0.40 & 0.74 & \\
\hline 4 & 0.48 & 0.52 & & & & \\
\hline Average & 0.31 & 0.53 & & & & \\
\hline Standard deviation & 0.17 & 0.24 & & & & \\
\hline
\end{tabular}




\section{Conclusion}

To improve the fabrication of dental implant surgical guides, a fast and accurate method was proposed in this research. The method streamlined the fabrication procedure by integrating dental implant software and a computer-controlled drilling machine. Pilot holes could then be automatically drilled on a dental implant surgical guide made of a vacuum-formed template. The marker plate with three ceramic balls and the specially-designed fixture saved measurement time and reduced measurement error. The fabrication time for a surgical guide using the method presented in the paper was under 30 minutes, considerably less fabrication time than that required to make a stereolithographic surgical guide. This method also made reworking on the surgical guides possible. However, the learning curve of the proposed method is steeper than that using 3D printers, and it is not as automatic. According to the clinical study, out of four patients with eight implants in total, the deviation between the planned and the actual implants was $0.31 \pm 0.17 \mathrm{~mm}$ in position and $0.53 \pm 0.24^{\circ}$ in orientation. Compared to the data found in the literature, it could be concluded that the use of the surgical guides fabricated by the proposed method in the paper appeared to be a feasible and promising technique for dental implantation.

\section{Acknowledgement}

The authors thank Kan-Shan Shih, DDS, for providing the clinical information.

\section{References}

[1] Y.J. Liu, S.M. Cui, C. He, J.K. Li and Q.Y. Wang, High cycle fatigue behavior of implant Ti-6Al-4V in air and simulated body fluid, Bio-Medical Materials and Engineering 24 (2014), 263-269.

[2] Y.M. Huang, I.C. Chou, C.P. Jiang, Y.S. Wu and S.Y. Lee, Finite element analysis of dental implant neck effects on primary stability and osseointegration in a type IV bone mandible, Bio-Medical Materials and Engineering 24 (2014), $1407-1415$.

[3] C.M. Becker and D.A. Kaiser, Surgical guide for dental implant placement, Journal of Prosthetic Dentistry 83 (2000), 248-251.

[4] S.S. Atsu, A surgical guide for dental implant placement in edentulous posterior regions, Journal of Prosthetic Dentistry 96 (2006), 129-133.

[5] N.K. Arfai and S. Kiat-amnuay, Radiographic and surgical guide for placement of multiple implants, Journal of Prosthetic Dentistry 97 (2007), 310-312.

[6] K. Akça, H. Iplikçioğlu and M.C. Çehreli, A surgical guide for accurate mesiodistal paralleling of implants in the posterior edentulous mandible, Journal of Prosthetic Dentistry 87 (2002), 233-235.

[7] P.Y.P. Wat, E.H.N. Pow, F.S.W. Chau and Leung K.C.M., A surgical guide for dental implant placement in an edentulous jaw, Journal of Prosthetic Dentistry 100 (2008), 323-325.

[8] F.M. George, H.L. Chan, M.E. Razzoog and T.J. Oh, Fabrication of a cast-based implant surgical guide using guide sleeves, Journal of Prosthetic Dentistry 106 (2011), 409-412.

[9] J.L. Shotwell, E.J. Billy, H.L. Wang and T.J. Oh, Implant surgical guide fabrication for partially edentulous patients, Journal of Prosthetic Dentistry 93 (2005), 294-297.

[10] A. Kleinman, F. Leyva, J. Lozada and R.D. Patel, Loma linda guide: A stereolithographically designed surgical template: Technique paper, The Journal of Oral Implantology 35 (2009), 238-244.

[11] G.D. Giacomo, J. Silva, R. Martines and S. Ajzen, Computer-designed selective laser sintering surgical guide and immediate loading dental implants with definitive prosthesis in edentulous patient: A preliminary method, European Journal of Dentistry 8 (2014), 100-106.

[12] D.P. Sarment, P. Sukovic and N. Clinthorne, Accuracy of implant placement with a stereolithographic surgical guide, International Journal of Oral and Maxillofacial Implants 18 (2003), 571-577. 